\title{
Synthesis and Properties of a Cationic Bidentate Lewis Acid
}

Min Hyung Lee and François P. Gabbaï*

Department of Chemistry, Texas A\&M University, College Station, Texas 77843, USA.

Fax: +1 979845 4719; Tel: +1 9798622070 .

francois@tamu.edu 
Optimized geometries of 3 and $[4]^{+}$

\begin{tabular}{|c|c|c|c|c|}
\hline \multicolumn{5}{|c|}{ Compound 3} \\
\hline \multicolumn{5}{|c|}{-2.135257} \\
\hline 2 & C & -2.982923 & -0.726566 & -0.138870 \\
\hline 3 & C & -4.370724 & -0.459713 & -0.122885 \\
\hline 4 & $\mathrm{H}$ & -4.707037 & 0.556526 & 0.107917 \\
\hline 5 & C & -5.352383 & -1.456303 & -0.367093 \\
\hline 6 & $\mathrm{H}$ & -6.415276 & -1.199586 & -0.312535 \\
\hline 7 & C & -4.950055 & -2.744780 & -0.681870 \\
\hline 8 & $\mathrm{H}$ & -5.687920 & -3.531390 & -0.879119 \\
\hline 9 & C & -3.181348 & -4.398931 & -1.160146 \\
\hline 10 & $\mathrm{H}$ & -3.971323 & -5.135456 & -1.347838 \\
\hline 11 & C & -1.844506 & -4.741178 & -1.292903 \\
\hline 12 & $\mathrm{H}$ & -1.552918 & -5.756848 & -1.580954 \\
\hline 13 & C & -0.846189 & -3.752861 & -1.066415 \\
\hline 14 & $\mathrm{H}$ & 0.201475 & -4.051023 & -1.198763 \\
\hline 15 & C & -1.156475 & -2.430044 & -0.697393 \\
\hline 16 & C & -2.543872 & -2.061106 & -0.523727 \\
\hline 17 & C & -3.561360 & -3.073271 & -0.784854 \\
\hline 18 & C & -1.071907 & 0.400578 & 1.590352 \\
\hline 19 & C & 0.030681 & 1.332369 & 1.672482 \\
\hline 20 & C & 0.877898 & 1.342546 & 2.800045 \\
\hline 21 & $\mathrm{H}$ & 1.723669 & 2.040529 & 2.815908 \\
\hline 22 & C & 0.671248 & 0.487788 & 3.900620 \\
\hline 23 & C & -0.401399 & -0.423766 & 3.828421 \\
\hline 24 & $\mathrm{H}$ & -0.581133 & -1.101152 & 4.672398 \\
\hline 25 & C & -1.258414 & -0.497262 & 2.709807 \\
\hline 26 & C & 0.355241 & 2.342701 & 0.580073 \\
\hline 27 & $\mathrm{H}$ & 1.435654 & 2.568317 & 0.583044 \\
\hline 28 & $\mathrm{H}$ & -0.193720 & 3.290379 & 0.735074 \\
\hline 29 & $\mathrm{H}$ & 0.092223 & 1.987018 & -0.427584 \\
\hline 30 & C & 1.557431 & 0.561511 & 5.128443 \\
\hline 31 & $\mathrm{H}$ & 1.597028 & -0.406402 & 5.657906 \\
\hline 32 & $\mathrm{H}$ & 1.178115 & 1.313622 & 5.847930 \\
\hline 33 & $\mathrm{H}$ & 2.588925 & 0.854195 & 4.865014 \\
\hline 34 & C & -2.378541 & -1.523644 & 2.783981 \\
\hline 35 & $\mathrm{H}$ & -2.428217 & -1.957261 & 3.797864 \\
\hline 36 & $\mathrm{H}$ & -2.218886 & -2.350832 & 2.070329 \\
\hline 37 & $\mathrm{H}$ & -3.366911 & -1.093116 & 2.549586 \\
\hline 38 & C & -2.579249 & 1.921283 & -0.135188 \\
\hline 39 & C & -2.538689 & 2.208797 & -1.541091 \\
\hline 40 & C & -2.938446 & 3.472079 & -2.020331 \\
\hline 41 & $\mathrm{H}$ & -2.876325 & 3.674429 & -3.097251 \\
\hline 42 & C & -3.425891 & 4.476419 & -1.156944 \\
\hline 43 & C & -3.493227 & 4.183404 & 0.218899 \\
\hline 44 & $\mathrm{H}$ & -3.882730 & 4.943568 & 0.907817 \\
\hline 45 & C & -3.072152 & 2.940628 & 0.743083 \\
\hline 46 & C & -2.045113 & 1.181462 & -2.549340 \\
\hline 47 & $\mathrm{H}$ & -1.055100 & 0.767115 & -2.277812 \\
\hline 48 & $\mathrm{H}$ & -1.950360 & 1.631214 & -3.553251 \\
\hline 49 & $\mathrm{H}$ & -2.734582 & 0.321369 & -2.623767 \\
\hline 50 & C & -3.880379 & 5.817855 & -1.700733 \\
\hline 51 & $\mathrm{H}$ & -4.094201 & 6.530871 & -0.886073 \\
\hline 52 & $\mathrm{H}$ & -4.801618 & 5.715808 & -2.306282 \\
\hline
\end{tabular}




\begin{tabular}{|c|c|c|c|c|}
\hline 53 & $\mathrm{H}$ & -3.112929 & 6.270258 & -2.355748 \\
\hline 54 & C & -3.186366 & 2.728037 & 2.247091 \\
\hline 55 & $\mathrm{H}$ & -3.769330 & 3.543571 & 2.709753 \\
\hline 56 & $\mathrm{H}$ & -2.194790 & 2.703204 & 2.734371 \\
\hline 57 & $\mathrm{H}$ & -3.687176 & 1.775366 & 2.498021 \\
\hline 58 & C & 2.574738 & -0.376671 & -0.576692 \\
\hline 59 & C & 3.491885 & -0.691789 & 0.466880 \\
\hline 60 & C & 4.804403 & -0.179850 & 0.448200 \\
\hline 61 & $\mathrm{H}$ & 5.478207 & -0.455675 & 1.263087 \\
\hline 62 & C & 5.258629 & 0.667054 & -0.601090 \\
\hline 63 & C & 4.347340 & 0.946346 & -1.656281 \\
\hline 64 & $\mathrm{H}$ & 4.657654 & 1.563767 & -2.502756 \\
\hline 65 & C & 3.030367 & 0.442050 & -1.647792 \\
\hline 66 & C & 3.086419 & -1.617086 & 1.605378 \\
\hline 67 & $\mathrm{H}$ & 2.154383 & -1.274548 & 2.091888 \\
\hline 68 & $\mathrm{H}$ & 3.869769 & -1.669100 & 2.382457 \\
\hline 69 & $\mathrm{H}$ & 2.913241 & -2.649256 & 1.241632 \\
\hline 70 & C & 2.114053 & 0.803825 & -2.807216 \\
\hline 71 & $\mathrm{H}$ & 2.679473 & 1.245142 & -3.647235 \\
\hline 72 & $\mathrm{H}$ & 1.348662 & 1.542983 & -2.500752 \\
\hline 73 & $\mathrm{H}$ & 1.577990 & -0.083686 & -3.193543 \\
\hline 74 & $\mathrm{Hg}$ & 0.610088 & -1.218419 & -0.547827 \\
\hline 75 & $\mathrm{~N}$ & 6.554964 & 1.218046 & -0.587414 \\
\hline 76 & C & 7.551122 & 0.622974 & 0.314830 \\
\hline 77 & $\mathrm{H}$ & 7.228842 & 0.721779 & 1.365813 \\
\hline 78 & $\mathrm{H}$ & 7.739135 & -0.454021 & 0.109165 \\
\hline 79 & $\mathrm{H}$ & 8.499510 & 1.172427 & 0.206260 \\
\hline 80 & C & 7.079352 & 1.785943 & -1.837426 \\
\hline 81 & $\mathrm{H}$ & 6.447959 & 2.626246 & -2.173977 \\
\hline 82 & $\mathrm{H}$ & 8.088589 & 2.184703 & -1.648784 \\
\hline 83 & $\mathrm{H}$ & 7.139302 & 1.043710 & -2.663990 \\
\hline \multicolumn{5}{|c|}{ 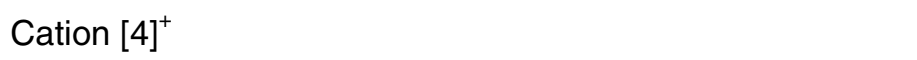 } \\
\hline 1 & B & -2.301433 & 0.522813 & 0.349945 \\
\hline 2 & C & -3.154404 & -0.686992 & -0.205152 \\
\hline 3 & C & -4.535778 & -0.388157 & -0.258858 \\
\hline 4 & $\mathrm{H}$ & -4.856947 & 0.640885 & -0.068731 \\
\hline 5 & C & -5.530260 & -1.366801 & -0.521049 \\
\hline 6 & $\mathrm{H}$ & -6.586992 & -1.083024 & -0.521590 \\
\hline 7 & C & -5.147777 & -2.673404 & -0.781402 \\
\hline 8 & $\mathrm{H}$ & -5.896174 & -3.446078 & -0.990051 \\
\hline 9 & C & -3.402104 & -4.384675 & -1.127078 \\
\hline 10 & $\mathrm{H}$ & -4.201028 & -5.106883 & -1.328250 \\
\hline 11 & C & -2.070714 & -4.767185 & -1.187728 \\
\hline 12 & $\mathrm{H}$ & -1.794862 & -5.798069 & -1.430737 \\
\hline 13 & C & -1.057456 & -3.798630 & -0.946219 \\
\hline 14 & $\mathrm{H}$ & -0.013148 & -4.125719 & -1.020963 \\
\hline 15 & C & -1.358662 & -2.460209 & -0.632821 \\
\hline 16 & C & -2.737030 & -2.043616 & -0.532121 \\
\hline 17 & C & -3.764750 & -3.039118 & -0.809742 \\
\hline 18 & C & -1.224495 & 0.422141 & 1.522618 \\
\hline 19 & C & -0.118449 & 1.351833 & 1.602963 \\
\hline 20 & C & 0.707863 & 1.388777 & 2.746226 \\
\hline 21 & $\mathrm{H}$ & 1.541284 & 2.102315 & 2.771079 \\
\hline 22 & C & 0.476798 & 0.563733 & 3.865759 \\
\hline 23 & C & -0.592524 & -0.352541 & 3.791847 \\
\hline
\end{tabular}




\begin{tabular}{|c|c|c|c|c|}
\hline 24 & $\mathrm{H}$ & -0.793804 & -1.003567 & 4.650952 \\
\hline 25 & C & -1.431920 & -0.450072 & 2.661173 \\
\hline 26 & C & 0.212981 & 2.350296 & 0.500740 \\
\hline 27 & $\mathrm{H}$ & 1.289313 & 2.598850 & 0.524222 \\
\hline 28 & $\mathrm{H}$ & -0.353556 & 3.290470 & 0.630685 \\
\hline 29 & $\mathrm{H}$ & -0.033328 & 1.979370 & -0.506472 \\
\hline 30 & C & 1.321393 & 0.682174 & 5.118879 \\
\hline 31 & $\mathrm{H}$ & 1.372846 & -0.274215 & 5.667394 \\
\hline 32 & $\mathrm{H}$ & 0.892758 & 1.431550 & 5.812319 \\
\hline 33 & $\mathrm{H}$ & 2.351379 & 1.006815 & 4.888052 \\
\hline 34 & C & -2.562631 & -1.462511 & 2.744175 \\
\hline 35 & $\mathrm{H}$ & -2.642511 & -1.860185 & 3.770047 \\
\hline 36 & $\mathrm{H}$ & -2.395527 & -2.315672 & 2.063342 \\
\hline 37 & $\mathrm{H}$ & -3.539562 & -1.030057 & 2.470628 \\
\hline 38 & C & -2.717497 & 1.956817 & -0.195530 \\
\hline 39 & C & -2.668508 & 2.239901 & -1.602171 \\
\hline 40 & C & -3.033691 & 3.511911 & -2.084553 \\
\hline 41 & $\mathrm{H}$ & -2.969195 & 3.710366 & -3.161892 \\
\hline 42 & C & -3.496983 & 4.530356 & -1.223084 \\
\hline 43 & C & -3.573014 & 4.241595 & 0.153843 \\
\hline 44 & $\mathrm{H}$ & -3.948886 & 5.011116 & 0.839173 \\
\hline 45 & C & -3.184643 & 2.989988 & 0.681483 \\
\hline 46 & C & -2.214004 & 1.194677 & -2.612397 \\
\hline 47 & $\mathrm{H}$ & -1.302290 & 0.656837 & -2.287056 \\
\hline 48 & $\mathrm{H}$ & -1.997696 & 1.661330 & -3.589536 \\
\hline 49 & $\mathrm{H}$ & -2.987750 & 0.421605 & -2.771623 \\
\hline 50 & C & -3.914962 & 5.881577 & -1.769684 \\
\hline 51 & $\mathrm{H}$ & -4.179546 & 6.580684 & -0.958652 \\
\hline 52 & $\mathrm{H}$ & -4.794942 & 5.790498 & -2.434219 \\
\hline 53 & $\mathrm{H}$ & -3.107671 & 6.344413 & -2.367225 \\
\hline 54 & C & -3.313775 & 2.779625 & 2.184475 \\
\hline 55 & $\mathrm{H}$ & -3.887155 & 3.603613 & 2.642751 \\
\hline 56 & $\mathrm{H}$ & -2.327567 & 2.741382 & 2.682647 \\
\hline 57 & $\mathrm{H}$ & -3.834060 & 1.836573 & 2.431583 \\
\hline 58 & C & 2.413187 & -0.482896 & -0.472238 \\
\hline 59 & C & 3.313593 & -0.806839 & 0.580862 \\
\hline 60 & C & 4.661980 & -0.369933 & 0.534988 \\
\hline 61 & $\mathrm{H}$ & 5.318765 & -0.640069 & 1.363785 \\
\hline 62 & C & 5.109951 & 0.379003 & -0.563253 \\
\hline 63 & C & 4.242407 & 0.696529 & -1.622293 \\
\hline 64 & $\mathrm{H}$ & 4.581141 & 1.268821 & -2.492093 \\
\hline 65 & C & 2.895845 & 0.266202 & -1.584613 \\
\hline 66 & C & 2.858629 & -1.636359 & 1.767878 \\
\hline 67 & $\mathrm{H}$ & 2.030745 & -1.137915 & 2.304292 \\
\hline 68 & $\mathrm{H}$ & 3.675636 & -1.805661 & 2.490445 \\
\hline 69 & $\mathrm{H}$ & 2.493664 & -2.628743 & 1.442166 \\
\hline 70 & C & 1.989866 & 0.612745 & -2.752197 \\
\hline 71 & $\mathrm{H}$ & 2.538823 & 1.119275 & -3.564523 \\
\hline 72 & $\mathrm{H}$ & 1.171069 & 1.283647 & -2.434841 \\
\hline 73 & $\mathrm{H}$ & 1.522913 & -0.294671 & -3.177491 \\
\hline 74 & C & 7.386102 & 0.461095 & 0.554127 \\
\hline 75 & $\mathrm{H}$ & 6.942332 & 0.896059 & 1.459764 \\
\hline 76 & $\mathrm{H}$ & 8.403317 & 0.852322 & 0.410457 \\
\hline 77 & $\mathrm{H}$ & 7.414788 & -0.634244 & 0.628243 \\
\hline 78 & C & 7.220297 & 0.261949 & -1.894704 \\
\hline 79 & $\mathrm{H}$ & 7.185092 & -0.832875 & -1.807001 \\
\hline
\end{tabular}




$\begin{array}{lllll}80 & \mathrm{H} & 8.261467 & 0.615076 & -1.945431 \\ 81 & \mathrm{H} & 6.668612 & 0.578605 & -2.788529 \\ 82 & \mathrm{C} & 6.583343 & 2.388373 & -0.749897 \\ 83 & \mathrm{H} & 6.095951 & 2.796906 & 0.146179 \\ 84 & \mathrm{H} & 6.034970 & 2.704919 & -1.645818 \\ 85 & \mathrm{H} & 7.630885 & 2.720663 & -0.808728 \\ 86 & \mathrm{~N} & 6.555864 & 0.862466 & -0.655056 \\ 87 & \mathrm{Hg} & 0.388917 & -1.259585 & -0.430657\end{array}$

UV-vis fluoride titration data obtained for $\mathbf{3}$ and $[\mathbf{4}]^{+}$in THF
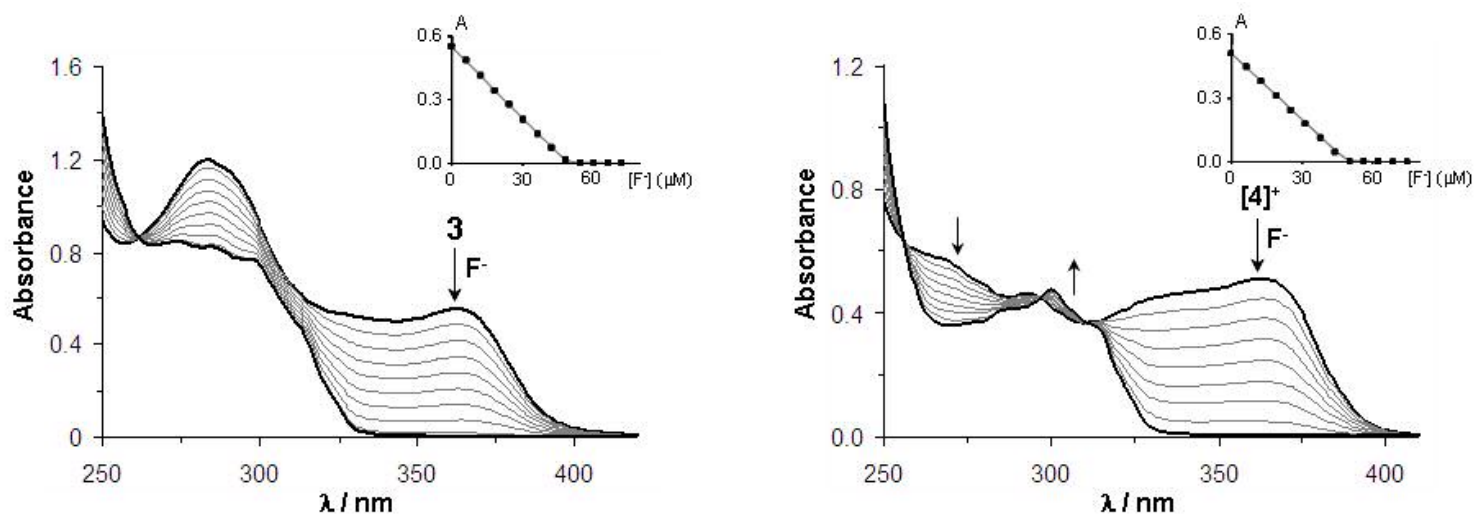

Changes in the UV-vis absorption spectra of a solution of 3 and [4][OTf] ( $3 \mathrm{~mL}, 5 \times 10^{-5}$ $\mathrm{M}$ in THF) upon addition of a TBAF solution in THF. See experimental in main text. 